TRANSACTIONS OF THE

AMERICAN MATHEMATICAL SOCIETY

Volume 360, Number 10, October 2008, Pages 5571-5588

S 0002-9947(08)04535-2

Article electronically published on May 20, 2008

\title{
ASYMPTOTIC ZERO DISTRIBUTION FOR A CLASS OF MULTIPLE ORTHOGONAL POLYNOMIALS
}

\author{
E. COUSSEMENT, J. COUSSEMENT, AND W. VAN ASSCHE
}

\begin{abstract}
We establish the asymptotic zero distribution for polynomials generated by a four-term recurrence relation with varying recurrence coefficients having a particular limiting behavior. The proof is based on ratio asymptotics for these polynomials. We can apply this result to three examples of multiple orthogonal polynomials, in particular Jacobi-Piñeiro, Laguerre I and the example associated with modified Bessel functions. We also discuss an application to Toeplitz matrices.
\end{abstract}

\section{INTRODUCTION}

Let $\mu$ be a positive measure on the real line for which the support is not finite and all the moments exist. The corresponding monic orthogonal polynomial $P_{n}$ of degree $n$ is then defined by

$$
\int x^{m} P_{n}(x) \mathrm{d} \mu(x)=0, \quad k=0, \ldots, n-1,
$$

with $P_{0} \equiv 1$ and $P_{-1} \equiv 0$. A well-known fact is that such polynomials satisfy a three-term recurrence relation of the form

$$
z P_{n}(z)=P_{n+1}(z)+b_{n} P_{n}(z)+a_{n}^{2} P_{n-1}(z), \quad a_{n}>0, b_{n} \in \mathbb{R},
$$

with initial conditions $P_{0} \equiv 1$ and $P_{-1} \equiv 0$.

An object of frequent study is the asymptotic zero distribution of the zeros for a sequence of orthogonal polynomials. The zeros of the polynomials $P_{n}$, generated by (1.2), are real and simple [13]. With each polynomial $P_{n}$ we can associate the normalized zero counting measure

$$
\nu\left(P_{n}\right):=\frac{1}{n} \sum_{P_{n}(x)=0} \delta_{x},
$$

where $\delta_{x}$ is the Dirac point mass at $x$. If $\lim _{n \rightarrow \infty} \nu\left(P_{n}\right)=\nu$, by which we mean that

$$
\lim _{n \rightarrow \infty} \int f \mathrm{~d} \nu\left(P_{n}\right)=\int f \mathrm{~d} \nu
$$

Received by the editors June 19, 2006 and, in revised form, January 31, 2007.

2000 Mathematics Subject Classification. Primary 33C45, 42C05; Secondary 15A18.

Key words and phrases. Multiple orthogonal polynomials, asymptotics.

This work was supported by INTAS project 03-51-6637, by FWO projects G.0455.04 and G.0184.02 and by OT/04/21 of Katholieke Universiteit Leuven.

The second author is a postdoctoral researcher at the Katholieke Universiteit Leuven (Belgium).

(C)2008 American Mathematical Society Reverts to public domain 28 years from publication 


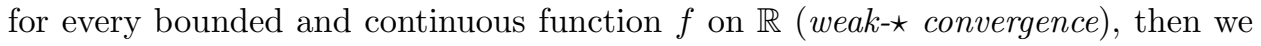
call the probability measure $\nu$ the asymptotic zero distribution of the sequence $\left\{P_{n}\right\}_{n \geq 0}$. One of the famous results in this context is the following.

Theorem 1.1 (see, e.g., [24, 28]). Suppose that the recurrence coefficients $a_{n}>0$ and $b_{n} \in \mathbb{R}$ have the limits $a>0$ and $b \in \mathbb{R}$, respectively. The polynomials $P_{n}$, generated by (1.2), then have the asymptotic zero distribution $\omega_{[\gamma, \delta]}$ with density

$$
\frac{\mathrm{d} \omega_{[\gamma, \delta]}}{\mathrm{d} x}(x)= \begin{cases}\frac{1}{\pi \sqrt{(\delta-x)(x-\gamma)}}, & x \in[\gamma, \delta], \\ 0, & \text { elsewhere, }\end{cases}
$$

where $\gamma=b-2 a$ and $\delta=b+2 a$.

Remark 1.2. The measure $\omega_{[\gamma, \delta]}$ is known as the arcsine measure on $[\gamma, \delta]$. It also minimizes the logarithmic energy of the interval $[\gamma, \delta][26$.

Recently, the result in Theorem 1.1 was extended to the case of varying recurrence coefficients. Here the notation $\lim _{n / N \rightarrow t} Y_{n, N}=Y$ denotes the property that in the doubly indexed sequence $Y_{n, N}$ we have $\lim _{j \rightarrow \infty} Y_{n_{j}, N_{j}}=Y$ whenever $n_{j}$ and $N_{j}$ are two sequences of natural numbers such that $N_{j} \rightarrow \infty$ and $n_{j} / N_{j} \rightarrow t$ as $j \rightarrow \infty$.

Theorem 1.3 (Kuijlaars, Van Assche [21]). Let for each $N \in \mathbb{N}$, two sequences $\left\{a_{n, N}\right\}_{n=1}^{\infty}, a_{n, N}>0$, and $\left\{b_{n, N}\right\}_{n=0}^{\infty}, b_{n, N} \in \mathbb{R}$, of recurrence coefficients be given. Furthermore, suppose there exist two continuous functions $a:(0,+\infty) \rightarrow[0,+\infty)$, $b:(0,+\infty) \rightarrow \mathbb{R}$, such that

$$
\lim _{n / N \rightarrow t} a_{n, N}=a(t), \quad \lim _{n / N \rightarrow t} b_{n, N}=b(t), \quad t>0,
$$

and define $\gamma(t):=b(t)-2 a(t), \delta:=b(t)+2 a(t), t>0$. For the (orthogonal) polynomials generated by the recurrence

$$
z P_{n, N}(z)=P_{n+1, N}(z)+b_{n, N} P_{n, N}(z)+a_{n, N}^{2} P_{n-1, N}(z)
$$

with initial conditions $P_{0, N} \equiv 1$ and $P_{-1, N} \equiv 0$, we then have

$$
\lim _{n / N \rightarrow t} \nu\left(P_{n, N}\right)=\frac{1}{t} \int_{0}^{t} \omega_{[\gamma(s), \delta(s)]} \mathrm{d} s, \quad t>0 .
$$

Here $\omega_{[\gamma, \delta]}$ is defined by (1.4) if $\gamma<\delta$ and by $\delta_{\gamma}$ if $\gamma=\delta$.

Remark 1.4. More recently, Theorem 1.3 was generalized to measurable functions $a$ and $b$ [20].

In this paper we present a (conditional) theorem giving the asymptotic zero distribution for polynomials satisfying a four-term recurrence relation of the form

$$
z P_{n, N}(z)=P_{n+1, N}(z)+b_{n, N} P_{n, N}(z)+c_{n, N} P_{n-1, N}(z)+d_{n, N} P_{n-2, N}(z),
$$

where the varying recurrence coefficients have some particular limiting behavior. So, in a sense it extends Theorem 1.3. Such a four-term recurrence relation appears in the theory of multiple orthogonal polynomials of Type II. These are a generalization of orthogonal polynomials which arises naturally in Hermite-Padé approximation of a system of (Markov) functions [9, 10, 22. In particular, they satisfy orthogonality conditions with respect to several positive measures [2, 25, 29]. Some of their applications are situated in diophantine number theory, rational approximation, 
spectral and scattering problems for higher-order difference equations and some associated dynamical systems; see, e.g., [5, 11, 18, 27]. Recently they also appeared in random matrix theory for matrix ensembles with external source [3, 7, 8] and Wishard ensembles [6]. The particular limiting behavior which we are considering appears in the examples Jacobi-Piñeiro, Laguerre I [29] and the example associated with modified Bessel functions [30].

In Subsection 2.1 we state our main theorem. Next, in Subsection 2.2 and Subsection 2.3 we apply this result to the examples of multiple orthogonal polynomials mentioned above and some particular kind of Toeplitz matrices. In Section 3 we discuss a theorem on ratio asymptotics for monic polynomials satisfying the recurrence (1.8). This will be used to prove our main theorem in Section 4

\section{Statement of Results}

2.1. Main theorem. We will study doubly indexed sequences of polynomials $\left\{P_{n, N}\right\}$, generated by a four-term recurrence of the form

$$
z P_{n, N}(z)=P_{n+1, N}(z)+b_{n, N} P_{n, N}(z)+c_{n, N} P_{n-1, N}(z)+d_{n, N} P_{n-2, N}(z),
$$

with the initial conditions $P_{0, N} \equiv 1, P_{-1, N} \equiv 0$ and $P_{-2, N} \equiv 0$ and real recurrence coefficients. In particular, our main theorem gives an explicit expression for the asymptotic zero distributions

$$
\lim _{n / N \rightarrow t} \nu\left(P_{n, N}\right), \quad t>0,
$$

with some conditions on the zeros of the $P_{n, N}$ and some particular limiting behavior for the recurrence coefficients. As mentioned in the introduction the limit is taken over any sequence $\left\{\nu\left(P_{n_{j}, N_{j}}\right)\right\}_{j \geq 1}$ for which $n_{j} \rightarrow \infty, N_{j} \rightarrow \infty$ and $n_{j} / N_{j} \rightarrow x$ as $j \rightarrow \infty$. We will use this notation throughout the rest of this paper.

Theorem 2.1. Let for each $N \in \mathbb{N}$ three sequences $\left\{b_{n, N}\right\}_{n=0}^{\infty},\left\{c_{n, N}\right\}_{n=1}^{\infty}$ and $\left\{d_{n, N}\right\}_{n=2}^{\infty}$ of real recurrence coefficients be given and assume that there exists a continuous function $\alpha:[0,+\infty) \rightarrow[0,+\infty)$ such that, for $t>0$,

$$
\lim _{n / N \rightarrow t} b_{n, N}=3 \beta(t), \quad \lim _{n / N \rightarrow t} c_{n, N}=3 \beta(t)^{2}, \quad \lim _{n / N \rightarrow t} d_{n, N}=\beta(t)^{3},
$$

with $\beta(t)=\frac{4 \alpha(t)}{27}$. Let $P_{n, N}$ be the monic polynomials generated by the recurrence (2.1) and suppose these polynomials $P_{n, N}$ have real simple zeros $x_{1}^{n, N}<\ldots<x_{n}^{n, N}$ satisfying the interlacing property $x_{j}^{n+1, N}<x_{j}^{n, N}<x_{j+1}^{n+1, N}$, for all $n, N \in \mathbb{N}$, $j=1, \ldots, n$. Then

$$
\lim _{n / N \rightarrow t} \nu\left(P_{n, N}\right)=\frac{1}{t} \int_{0}^{t} v_{[0, \alpha(s)]} \mathrm{d} s, \quad t>0
$$

where $v_{[0, \alpha]}$ is defined by $\delta_{0}$ if $\alpha=0$ and $\frac{\mathrm{d} v_{[0, \alpha]}}{\mathrm{d} x}(x)=\frac{1}{\alpha} \frac{\mathrm{d} v_{[0,1]}}{\mathrm{d} x}\left(\frac{x}{\alpha}\right)$, with

$$
\frac{\mathrm{d} v_{[0,1]}}{\mathrm{d} x}(x)= \begin{cases}\frac{\sqrt{3}}{4 \pi} \frac{(1+\sqrt{1-x})^{1 / 3}+(1-\sqrt{1-x})^{1 / 3}}{x^{2 / 3} \sqrt{1-x}}, & x \in(0,1) \\ 0, & \text { elsewhere }\end{cases}
$$

if $\alpha>0$. 


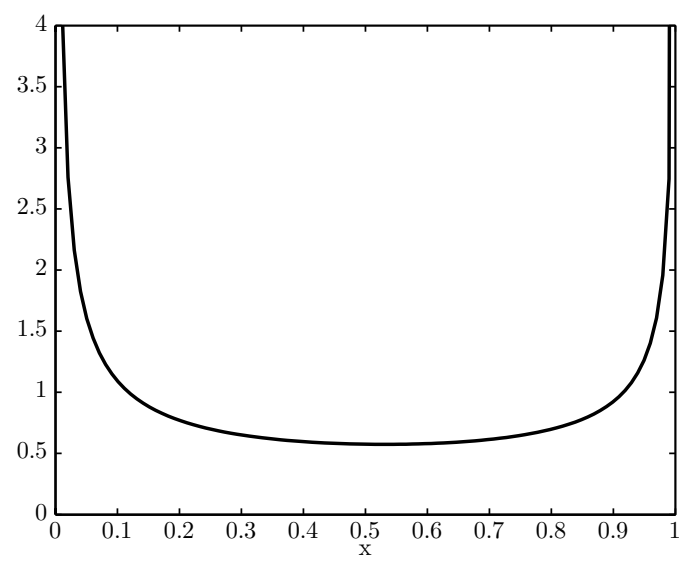

FIGURE 1. The density of the measure $v_{[0,1]}$.

Remark 2.2. Denote by $\nu_{t}$ the right hand side of (2.3). The action of this measure on arbitrary Borel sets is given by

$$
\nu_{t}(E)=\frac{1}{t} \int_{0}^{t} v_{[0, \alpha(s)]}(E) \mathrm{d} s, \quad E \in \mathcal{B}(\mathbb{R}) .
$$

Now suppose that for each $x \geq 0$ the set $\{s \geq 0 \mid x \leq \alpha(s)\}$ is an interval, which we denote by $\left[t_{-}(x), t_{+}(x)\right]$. The density of the measure $\nu_{t}$ is then

$$
\frac{\mathrm{d} \nu_{t}}{\mathrm{~d} x}(x)=\frac{1}{t} \int_{\min \left(t, t_{-}(x)\right)}^{\min \left(t, t_{+}(x)\right)} \frac{\mathrm{d} v_{[0, \alpha(s)]}}{\mathrm{d} x}(x) \mathrm{d} s .
$$

This will be the case in each of the examples we present in this paper.

Remark 2.3. Comparing Theorem 2.1 with Theorem 1.3 we see that the measure $v_{[0,1]}$ plays the role of the arcsine measure in the case of orthogonal polynomials (satisfying a three-term recurrence relation). The density (2.4) again has the behavior $c_{1}(1-x)^{-1 / 2}$ as $x \uparrow 1$, but has a different behavior $c_{2} x^{-2 / 3}$ as $x \downarrow 0$. See Figure 1.

Remark 2.4. The measure $v_{[0,1]}$ coincides (after a cubic transformation) with the asymptotic zero distribution of Faber polynomials associated with the 3-cusped hypocycloid [19].

2.2. Application to multiple orthogonal polynomials. There are two types of multiple orthogonal polynomials, but we will only consider type II. Let $\mu_{1}, \ldots, \mu_{r}$, $r \in \mathbb{N}$, be a set of positive measures on the real line for which the support is not finite and all the moments exist. Furthermore, let $\vec{n}=\left(n_{1}, n_{2}, \ldots, n_{r}\right)$ be a vector of $r$ nonnegative integers, which is a multi-index with length $|\vec{n}|:=n_{1}+n_{2}+\cdots+n_{r}$. A multiple orthogonal polynomial $P_{\vec{n}}$ of type II with respect to the multi-index $\vec{n}$, is a (nontrivial) polynomial of degree $\leq|\vec{n}|$ which satisfies the orthogonality conditions

$$
\int x^{m} P_{\vec{n}}(x) \mathrm{d} \mu_{j}(x)=0, \quad 0 \leq m \leq n_{j}-1, \quad j=1, \ldots, r .
$$


A basic requirement in the study of multiple orthogonal polynomials is that the system (2.6) has a unique solution (up to a scalar multiplicative constant) of degree $|\vec{n}|$. We call $\vec{n}$ a normal index for $\mu_{1}, \ldots, \mu_{r}$ if any solution of (2.6) has exactly degree $|\vec{n}|$ (which implies uniqueness). If all the multi-indices are normal, then the system of measures is called perfect. Some famous classes of perfect systems are the Angelesco systems, Nikishin systems (for $r=2$ ) and AT systems; see, e.g., 25, 29.

Multiple orthogonal polynomials of type II satisfy a recurrence relation of order $r+1$. In particular, if we set $r=2$ and consider proper multi-indices $\vec{\nu}_{n}=(m+s, m)$, $n \in \mathbb{N} \cup\{0\}$, where $n=2 m+s, s \in\{0,1\}$, then the polynomials $P_{n}:=P_{\vec{\nu}_{n}}$ satisfy a four-term recurrence relation of the form

$$
z P_{n}(z)=P_{n+1}(z)+b_{n} P_{n}(z)+c_{n} P_{n-1}(z)+d_{n} P_{n-2}(z),
$$

with the initial conditions $P_{0} \equiv 1, P_{-1} \equiv 0$ and $P_{-2} \equiv 0$. For three examples known in the literature the recurrence coefficients in (2.7) have the particular limiting behavior (2.2), possibly after some re-scaling. In each of these examples the measures form an AT system on an interval $\Delta \subseteq \mathbb{R}$. It is then known that the zeros of the polynomials $P_{n}$ are simple, lie in $\Delta[25,29]$ and satisfy the interlacing property [4. So, it is possible to apply Theorem 2.1.

2.2.1. Jacobi-Piñeiro. The Jacobi-Piñeiro polynomials are the multiple orthogonal polynomials for the system of orthogonality measures

$$
\mathrm{d} \mu_{j}(x)=x^{\alpha_{j}}(1-x)^{\beta} \mathrm{d} x, \quad j=1,2,
$$

on the interval $[0,1]$ with $\alpha_{1}, \alpha_{2}, \beta>-1$ and $\alpha_{2}-\alpha_{1} \notin \mathbb{Z}$. In [29] it was shown that the monic Jacobi-Piñeiro polynomials with respect to proper multi-indices, which we denote by $P_{n}^{\alpha_{1}, \alpha_{2} ; \beta}$, satisfy a recurrence relation of the form (2.7) for which

$$
\lim _{n \rightarrow \infty} b_{n}=3\left(\frac{4}{27}\right), \quad \lim _{n \rightarrow \infty} c_{n}=3\left(\frac{4}{27}\right)^{2}, \quad \lim _{n \rightarrow \infty} d_{n}=\left(\frac{4}{27}\right)^{3} .
$$

By Theorem 2.1 with $\alpha(t)=1, t>0$, we then easily obtain the following result.

Theorem 2.5. The Jacobi-Piñeiro polynomials $P_{n}^{\alpha_{1}, \alpha_{2} ; \beta}$ have the asymptotic zero distribution $v_{[0,1]}$, defined as in (2.4).

2.2.2. Multiple Laguerre I. The multiple Laguerre polynomials of the first kind are orthogonal with respect to the system of measures

$$
\mathrm{d} \mu_{j}(x)=x^{\alpha_{j}} \mathrm{e}^{-x} \mathrm{~d} x, \quad j=1,2,
$$

on $[0,+\infty)$ with $\alpha_{1}, \alpha_{2}>-1$ and $\alpha_{2}-\alpha_{1} \notin \mathbb{Z}$. Denote the monic multiple Laguerre I polynomials with respect to proper multi-indices by $L_{n}^{\alpha_{1}, \alpha_{2}}$. These satisfy a fourterm recurrence relation of the form (2.7) where, for $t>0$,

$$
\lim _{n / N \rightarrow t} \frac{b_{n}}{N}=3\left(\frac{t}{2}\right), \quad \lim _{n / N \rightarrow t} \frac{c_{n}}{N^{2}}=3\left(\frac{t}{2}\right)^{2}, \quad \lim _{n / N \rightarrow t} \frac{d_{n}}{N^{3}}=\left(\frac{t}{2}\right)^{3} ;
$$

see [29]. The following theorem is then a corollary of Theorem 2.1.

Theorem 2.6. For the multiple Laguerre polynomials of the first kind the limit

$$
\nu_{t}^{L}:=\lim _{n / N \rightarrow t} \frac{1}{n} \sum_{L_{n}^{\alpha_{1}}, \alpha_{2}(x)=0} \delta_{x / N}, \quad t>0
$$




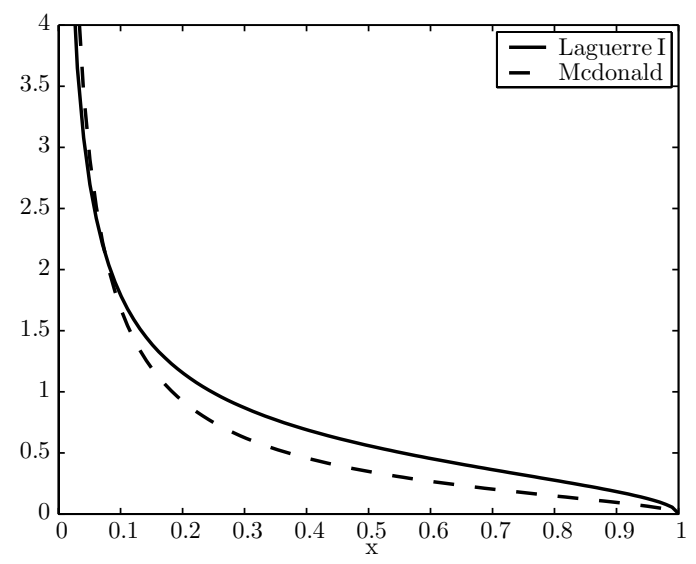

Figure 2. The densities of the measures $\nu_{t}^{L}$ and $\nu_{t}^{M}$, see (2.11) and (2.14), with $t=\frac{8}{27}$ and $t=\frac{2}{3 \sqrt{3}}$, respectively.

exists and has the density

$$
\frac{\mathrm{d} \nu_{t}^{L}}{\mathrm{~d} x}(x)= \begin{cases}\frac{8}{27 t} g\left(\frac{8 x}{27 t}\right), & x \in\left(0, \frac{27 t}{8}\right) \\ 0, & \text { elsewhere }\end{cases}
$$

where, for $y \in(0,1)$,

$$
g(y)=\frac{3 \sqrt{3}}{16 \pi} \frac{(1+3 \sqrt{1-y})(1-\sqrt{1-y})^{1 / 3}-(1-3 \sqrt{1-y})(1+\sqrt{1-y})^{1 / 3}}{y^{2 / 3}} .
$$

Proof. If we define $\tilde{L}_{n}^{\alpha_{1}, \alpha_{2}}(z):=L_{n}^{\alpha_{1}, \alpha_{2}}(N z) / N^{n}$, then the polynomials satisfy a recurrence relation of the form (2.1) and the asymptotic property (2.2) with $\alpha(t)=$ $\frac{27 t}{8}, t>0$. So, applying Theorem 2.1 we get

$$
\frac{\mathrm{d} \nu_{t}^{L}}{\mathrm{~d} x}(x)=\frac{1}{t} \int_{\frac{8 x}{27}}^{t} \frac{8}{27 s} \frac{\mathrm{d} v_{[0,1]}}{\mathrm{d} x}\left(\frac{8 x}{27 s}\right) \mathrm{d} s=\frac{1}{t} \frac{8}{27} \int_{\frac{\delta x}{27 t}}^{1} \frac{1}{u} \frac{\mathrm{d} v_{[0,1]}}{\mathrm{d} x}(u) \mathrm{d} u .
$$

Set $x \in\left(0, \frac{27 t}{8}\right)$ and $z=\sqrt{1-\frac{8 x}{27 t}}$. Applying the substitution $y \leftrightarrow \sqrt{1-u}$ we then obtain

$$
\begin{aligned}
\frac{\mathrm{d} \nu_{t}^{L}}{\mathrm{~d} x}(x) & =\frac{1}{t} \frac{4 \sqrt{3}}{27 \pi} \int_{0}^{z}\left((1+y)^{-4 / 3}(1-y)^{-5 / 3}+(1+y)^{-5 / 3}(1-y)^{-4 / 3}\right) \mathrm{d} y \\
& =\frac{1}{t} \frac{4 \sqrt{3}}{27 \pi} \int_{-z}^{z}(1+y)^{-5 / 3}(1-y)^{-4 / 3} \mathrm{~d} y \\
& =\left.\frac{1}{t} \frac{\sqrt{3}}{18 \pi} \frac{1+3 y}{(1+y)^{2 / 3}(1-y)^{1 / 3}}\right|_{-z} ^{z} .
\end{aligned}
$$

This completes the proof. 
2.2.3. Multiple orthogonal polynomials associated with modified Bessel functions. In [30] one considered multiple orthogonal polynomials with respect to the orthogonality measures

$$
\mathrm{d} \mu_{1}(x)=x^{\kappa} \rho_{\gamma}(x) \mathrm{d} x, \quad \mathrm{~d} \mu_{2}(x)=x^{\kappa} \rho_{\gamma+1}(x) \mathrm{d} x
$$

on $(0,+\infty)$ with $\kappa>-1, \gamma \geq 0$ and

$$
\rho_{\gamma}(x)=2 x^{\gamma / 2} K_{\gamma}(2 \sqrt{x}), \quad x>0,
$$

where $K_{\gamma}$ is the modified Bessel of the second kind, also known as the Macdonald function [1, p. 374]. For the type II multiple polynomials with respect to proper multi-indices, $P_{n}^{\gamma ; \kappa}$, the recurrence coefficients in (2.7) are known [30, Theorem 4]. In particular, for $t>0$,

$$
\lim _{n / N \rightarrow t} \frac{b_{n}}{N^{2}}=3 t^{2}, \quad \lim _{n / N \rightarrow t} \frac{c_{n}}{N^{4}}=3 t^{4}, \quad \lim _{n / N \rightarrow t} \frac{d_{n}}{N^{6}}=t^{6} .
$$

Theorem 2.1 then implies the following asymptotic result for the zeros of these polynomials.

Theorem 2.7. For the multiple orthogonal polynomials associated with modified Bessel functions the limit

$$
\nu_{t}^{M}:=\lim _{n / N \rightarrow t} \frac{1}{n} \sum_{P_{n}^{\gamma ; \kappa}(x)=0} \delta_{x / N^{2}}
$$

exists and has the density

$$
\frac{\mathrm{d} \nu_{t}^{M}}{\mathrm{~d} x}(x)= \begin{cases}\frac{4}{27 t^{2}} h\left(\frac{4 x}{27 t^{2}}\right), & x \in\left(0, \frac{27 t^{2}}{4}\right), \\ 0, & \text { elsewhere }\end{cases}
$$

where

$$
h(y)=\frac{3 \sqrt{3}}{4 \pi} \frac{(1+\sqrt{1-y})^{1 / 3}-(1-\sqrt{1-y})^{1 / 3}}{y^{2 / 3}}, \quad y \in(0,1) .
$$

Proof. Define the polynomials $\tilde{P}_{n}^{\gamma ; \kappa}(z):=P_{n}^{\gamma ; \kappa}\left(N^{2} z\right) / N^{2 n}$. By (2.12) these satisfy a recurrence relation of the form (2.1) having the asymptotic property (2.2) with $\alpha(t)=\frac{27 t^{2}}{4}, t>0$. Set $x \in\left(0, \frac{27 t^{2}}{4}\right)$ and $z=\frac{4 x}{27 t^{2}}$. Applying Theorem 2.1 then gives

$$
\frac{\mathrm{d} \nu_{t}^{M}}{\mathrm{~d} x}(x)=\frac{1}{t} \int_{\frac{2}{3} \sqrt{\frac{x}{3}}}^{t} \frac{4}{27 s^{2}} \frac{\mathrm{d} v_{[0,1]}}{\mathrm{d} x}\left(\frac{4 x}{27 s^{2}}\right) \mathrm{d} s=\frac{1}{t^{2}} \frac{2}{27 \sqrt{z}} \int_{z}^{1} \frac{1}{\sqrt{u}} \frac{\mathrm{d} v_{[0,1]}}{\mathrm{d} x}(u) \mathrm{d} u .
$$

Similarly as in Theorem 2.6 we apply the substitution $y \leftrightarrow \sqrt{1-u}$ and get

$$
\begin{aligned}
\frac{\mathrm{d} \nu_{t}^{M}}{\mathrm{~d} x}(x) & =\frac{1}{t^{2}} \frac{\sqrt{3}}{27 \pi} \frac{1}{\sqrt{z}} \int_{-\sqrt{1-z}}^{\sqrt{1-z}}(1+y)^{-5 / 6}(1-y)^{-7 / 6} \mathrm{~d} y \\
& =\left.\frac{1}{t^{2}} \frac{1}{3 \sqrt{3} \pi} \frac{1}{\sqrt{z}}\left(\frac{1+y}{1-y}\right)^{1 / 6}\right|_{-\sqrt{1-z}} ^{\sqrt{1-z}} .
\end{aligned}
$$

From this we easily obtain (2.14). 
2.3. Application to Toeplitz matrices. Set $\alpha>0, \beta=\frac{4 \alpha}{27}$, and define the Toeplitz matrices

$$
T_{n}^{\alpha}:=\left(\begin{array}{cccccc}
3 \beta & 1 & 0 & \ldots & \ldots & 0 \\
3 \beta^{2} & 3 \beta & 1 & \ddots & & \vdots \\
\beta^{3} & 3 \beta^{2} & 3 \beta & 1 & \ddots & \vdots \\
0 & \beta^{3} & 3 \beta^{2} & 3 \beta & \ddots & 0 \\
\vdots & \ddots & \ddots & \ddots & \ddots & 1 \\
0 & \ldots & 0 & \beta^{3} & 3 \beta^{2} & 3 \beta
\end{array}\right) \in \mathbb{R}^{n \times n}, \quad n \in \mathbb{N} .
$$

Note that the eigenvalues of $T_{n}^{\alpha}$ coincide with the zeros of the monic polynomial $Q_{n}^{\alpha}(z)=\operatorname{det}\left(z I_{n}-T_{n}^{\alpha}\right), n \in \mathbb{N}$. These polynomials satisfy the recurrence relation

$$
z Q_{n}^{\alpha}(z)=Q_{n+1}^{\alpha}(z)+3 \beta Q_{n}^{\alpha}(z)+3 \beta^{2} Q_{n-1}^{\alpha}(z)+\beta^{3} Q_{n-2}^{\alpha}(z),
$$

with $Q_{0}^{\alpha} \equiv 1$ and $Q_{-1}^{\alpha} \equiv Q_{-2}^{\alpha} \equiv 0$. The following asymptotic result for the eigenvalues of the matrices $T_{n}^{\alpha}$ then follows from Theorem 2.1.

Theorem 2.8. The limiting eigenvalue distribution of the matrices $T_{n}^{\alpha}$, with $\alpha>0$, is given by the measure $v_{[0, \alpha]}$, defined as in Theorem 2.1]

Proof. The homogeneous recurrence relation

$$
0=Q_{n+1}^{\alpha}(0)+3 \beta Q_{n}^{\alpha}(0)+3 \beta^{2} Q_{n-1}^{\alpha}(0)+\beta^{3} Q_{n-2}^{\alpha}(0)
$$

with $Q_{0}^{\alpha}(0)=1$ and $Q_{-1}^{\alpha}(0)=Q_{-2}^{\alpha}(0)=0$ has the solution

$$
Q_{n}^{\alpha}(0)=(-\beta)^{n}\left(1+\frac{3 n}{2}+\frac{n^{2}}{2}\right), \quad n \in \mathbb{N}
$$

So, since $\beta=\frac{4 \alpha}{27}>0$ all the $T_{n}^{\alpha}$ are nonsingular. Next, define

$$
\tilde{T}_{n}^{\alpha}=\left(\begin{array}{ccccccc}
1 & 0 & \ldots & \ldots & \ldots & \ldots & 0 \\
3 \beta & 1 & \ddots & & & & \vdots \\
3 \beta^{2} & 3 \beta & 1 & \ddots & & & \vdots \\
\beta^{3} & 3 \beta^{2} & 3 \beta & 1 & \ddots & & \vdots \\
0 & \beta^{3} & 3 \beta^{2} & 3 \beta & 1 & \ddots & \vdots \\
\vdots & \ddots & \ddots & \ddots & \ddots & \ddots & 0 \\
0 & \ldots & 0 & \beta^{3} & 3 \beta^{2} & 3 \beta & 1
\end{array}\right) \in \mathbb{R}^{(n+1) \times(n+1)}
$$

and notice that $\tilde{T}_{n}^{\alpha}=\left(\tilde{A}_{n}^{\alpha}\right)^{3}$ with

$$
\tilde{A}_{n}^{\alpha}=\left(\begin{array}{ccccc}
1 & 0 & \ldots & \ldots & 0 \\
\beta & 1 & \ddots & & \vdots \\
0 & \beta & 1 & \ddots & \vdots \\
\vdots & \ddots & \ddots & \ddots & 0 \\
0 & \ldots & 0 & \beta & 1
\end{array}\right) \in \mathbb{R}^{(n+1) \times(n+1)}
$$

Since $\tilde{A}_{n}^{\alpha}$ is totally nonnegative, $\tilde{T}_{n}^{\alpha}$ is totally nonnegative, see, e.g., [15, p. 74, $1^{\circ}$ ], and so also $T_{n}^{\alpha}$. By [15, p. 100, Theorem 10] we then get that the $T_{n}^{\alpha}$ are oscillation 
matrices. Consequently, the zeros of the polynomials $Q_{n}$ are simple and positive [15, p. 87, Theorem 6] and satisfy the interlacing property [15, p. 107, Theorem 14]. The theorem then easily follows from Theorem 2.1 and the recurrence (2.15).

Remark 2.9. The polynomials satisfying the recurrence relation with constant coefficients (2.15) are the multiple Chebyshev polynomials of the second kind after a cubic transformation [12, 14. These are an example of multiple orthogonal polynomials of type II extending the well-known Chebyshev polynomials of the second kind [13. The corresponding orthogonality measures can be found in [12, Corollary 4.2], [14, Theorem 4.1].

\section{RATiO ASYMPTOTICS}

In 21] Kuijlaars and Van Assche have proven a theorem that gives explicit ratio asymptotics for orthogonal polynomials with converging varying recurrence coefficients. In this section we give an extension of this result to polynomials satisfying a (specific) four-term recurrence relation instead of a three-term recurrence relation.

Theorem 3.1. Suppose we have for each $N \in \mathbb{N}$ sequences $\left\{b_{n, N}\right\}_{n=0}^{\infty},\left\{c_{n, N}\right\}_{n=1}^{\infty}$ and $\left\{d_{n, N}\right\}_{n=2}^{\infty}$ of real recurrence coefficients and let $P_{n, N}$ be the monic polynomials generated by the recurrence

$$
z P_{n, N}(z)=P_{n+1, N}(z)+b_{n, N} P_{n, N}(z)+c_{n, N} P_{n-1, N}(z)+d_{n, N} P_{n-2, N}(z),
$$

with $P_{0, N} \equiv 1, P_{-1, N} \equiv 0$ and $P_{-2, N} \equiv 0$. Assume that for some fixed $t>0$ the recurrence coefficients have the limits

$$
\lim _{n / N \rightarrow t} b_{n, N}=3\left(\frac{4 \alpha}{27}\right), \quad \lim _{n / N \rightarrow t} c_{n, N}=3\left(\frac{4 \alpha}{27}\right)^{2}, \quad \lim _{n / N \rightarrow t} d_{n, N}=\left(\frac{4 \alpha}{27}\right)^{3},
$$

with $\alpha \geq 0$. Furthermore, assume that the polynomials $P_{n, N}$ have real simple zeros $x_{1}^{n, N}<\ldots<x_{n}^{n, N}$ satisfying the interlacing property $x_{j}^{n+1, N}<x_{j}^{n, N}<x_{j+1}^{n+1, N}$, for all $n, N \in \mathbb{N}, j=1, \ldots, n$. Moreover, suppose that for some $t^{*}>t$ there exist $m \leq 0, M \geq \alpha$ such that all zeros of $P_{n, N}$ belong to $[m, M]$ whenever $n \leq t^{*} N$. Then

$$
\lim _{n / N \rightarrow t} \frac{P_{n, N}(z)}{P_{n+1, N}(z)}= \begin{cases}\frac{1}{\alpha} \phi\left(\frac{z}{\alpha}\right), & \alpha>0, \\ \frac{1}{z}, & \alpha=0,\end{cases}
$$

uniformly on compact subsets of $\mathbb{C} \backslash[m, M]$, where $\phi$ is defined by

$$
\phi(z):=\frac{27}{4}\left(\frac{3 \omega_{3}}{2} z^{1 / 3}\left\{\omega_{3}(-1+\sqrt{1-z})^{1 / 3}+(-1-\sqrt{1-z})^{1 / 3}\right\}-1\right)
$$

with $\omega_{3}=e^{\frac{2 \pi i}{3}}$ and

$$
\begin{aligned}
& \sqrt{\rho e^{i \theta}}=\rho^{1 / 2} e^{\frac{i \theta}{2}}, \quad \rho>0, \quad \theta \in[0,2 \pi), \\
& \left(\rho e^{i \theta}\right)^{1 / 3}=\rho^{1 / 3} e^{\frac{i \theta}{3}}, \quad \rho>0, \quad \theta \in(-\pi,+\pi] .
\end{aligned}
$$

Remark 3.2. In the case that the recurrence coefficients do not depend on $N$ the existence of the limit (3.3) was already proven in [4. Our proof will be based on similar arguments. 
Remark 3.3. Under the conditions of Theorem 3.1, by taking the derivative of (3.3) we get

$$
\lim _{n / N \rightarrow t}\left(\frac{P_{n, N}^{\prime}(z)}{P_{n, N}(z)}-\frac{P_{n+1, N}^{\prime}(z)}{P_{n+1, N}(z)}\right)= \begin{cases}\frac{1}{\alpha} \frac{\phi^{\prime}(z / \alpha)}{\phi(z / \alpha)}, & \alpha>0, \\ -\frac{1}{z}, & \alpha=0,\end{cases}
$$

uniformly on compact subsets of $\mathbb{C} \backslash[m, M]$.

In order to prove Theorem 3.1 we need part (a) of the following lemma. The whole lemma will be used in the proof of Theorem 2.1 as well. It can be found in, e.g., [21, Lemma 2.2], but we include a short proof for completeness.

Lemma 3.4. Suppose that the zeros of the monic polynomials $p_{n-1}$ and $p_{n}$, with degree $n-1$ and $n$, respectively, are simple and real, interlace and lie in $[m, M]$. Then

(a) $\left|\frac{p_{n-1}(z)}{p_{n}(z)}\right| \leq \frac{1}{\operatorname{dist}(z,[m, M])}, \quad \forall z \in \mathbb{C} \backslash[m, M]$,

(b) $\left|\frac{p_{n-1}(z)}{p_{n}(z)}\right| \geq \frac{1}{2|z|}, \quad$ if $|z|>\max (|m|,|M|)$.

Proof. Denote the real zeros of $p_{n}$ by $y_{1}, \ldots, y_{n}$. Since $p_{n-1}$ and $p_{n}$ are monic and their zeros interlace, there exist $w_{j}>0, \sum_{j=1}^{n} w_{j}=1$, so that

$$
\frac{p_{n-1}(z)}{p_{n}(z)}=\sum_{j=1}^{n} \frac{w_{j}}{z-y_{j}} \text {. }
$$

Then note that, because $y_{j} \in[m, M]$, for all $z \in \mathbb{C} \backslash[m, M]$ we have $\left|z-y_{j}\right| \geq$ $\operatorname{dist}(z,[m, M]), 1 \leq j \leq n$. This immediately proves part (a) of the lemma.

If $|z|>\max (|m|,|M|)$, then $\left|y_{j} / z\right|<1$ and therefore $\Re\left(\frac{1}{1-y_{j} / z}\right)>\frac{1}{2}, 1 \leq j \leq n$. Hence

$$
\frac{1}{|z|}\left|\sum_{j=1}^{n} \frac{w_{j}}{1-y_{j} / z}\right| \geq \frac{1}{|z|} \Re\left(\sum_{j=1}^{n} \frac{w_{j}}{1-y_{j} / z}\right)>\frac{1}{2|z|} \sum_{j=1}^{n} w_{j}=\frac{1}{2|z|},
$$

which proves part (b).

We also need the following properties of the function $\phi$.

Lemma 3.5. The function $\phi$ is analytic on $\mathbb{C} \backslash[0,1]$ and satisfies
(a) $z \phi(z)=\left(1+\frac{4 \phi(z)}{27}\right)^{3}, \quad z \in \mathbb{C} \backslash[0,1]$,
(b) $\phi(z)=z^{-1}+\mathcal{O}\left(z^{-2}\right), \quad$ as $z \rightarrow \infty$.

Proof. By the choice of branch cuts for the square and the cubic root, see (3.5), the function $\phi$ is certainly analytic on $\mathbb{C} \backslash(-\infty, 1]$. For $x<0$, a simple calculation also shows that $\lim _{\epsilon \downarrow 0} \phi(x+i \epsilon)=\lim _{\epsilon \downarrow 0} \phi(x-i \epsilon)$. So $\phi$ is analytic on $\mathbb{C} \backslash[0,1]$. If we define

$$
u_{ \pm}(z):=-1 \pm \sqrt{1-z},
$$

then

$$
u_{+}(z) u_{-}(z)=z, \quad u_{+}(z)+u_{-}(z)=-2 .
$$


Using this gives

$$
\begin{aligned}
\left(1+\frac{4 \phi(z)}{27}\right)^{3} & =\frac{27}{8} z\left(\omega_{3}\left(u_{+}(z)\right)^{1 / 3}+\left(u_{-}(z)\right)^{1 / 3}\right)^{3} \\
& =\frac{27}{8} z\left(-2+3 \omega_{3} z^{1 / 3}\left(\omega_{3}\left(u_{+}(z)\right)^{1 / 3}+\left(u_{-}(z)\right)^{1 / 3}\right)\right),
\end{aligned}
$$

which verifies part (a) of the lemma.

Next, we will prove that $\phi$ tends to zero as $z \rightarrow \infty$. From part (a) we then obtain that $\lim _{z \rightarrow \infty} z \phi(z)=1$ and, since $\phi$ is analytic in a neighborhood of infinity, this implies part (b) of the lemma. Applying the formula $(a+b)\left(a^{2}+b^{2}-a b\right)=a^{3}+b^{3}$ and (3.8), we observe that

$$
\frac{4}{27} \phi(z)=-\frac{3 \omega_{3} z^{1 / 3}}{\omega_{3}^{2}\left[\left(u_{+}(z)\right)^{1 / 3}\right]^{2}+\left[\left(u_{-}(z)\right)^{1 / 3}\right]^{2}-\omega_{3} z^{1 / 3}}-1 .
$$

Now take for a moment $z=1+L$, with $L>0$. By the definition of the square root we have $u_{ \pm}(1+L)=-1 \pm i \sqrt{L}$. So we can write

$$
\begin{aligned}
& u_{+}(1+L)=\rho(L) \mathrm{e}^{i\left(\frac{\pi}{2}+\varepsilon(L)\right)}, \\
& u_{-}(1+L)=\rho(L) \mathrm{e}^{i\left(-\frac{\pi}{2}-\varepsilon(L)\right)}, \quad 0<\varepsilon(L)<\frac{\pi}{2} .
\end{aligned}
$$

Obviously we then get

$$
\begin{aligned}
\omega_{3}^{2}\left[\left(u_{+}(1+L)\right)^{1 / 3}\right]^{2} & =(\rho(L))^{2 / 3} \mathrm{e}^{i\left(-\frac{\pi}{3}+\frac{2}{3} \varepsilon(L)\right)}, \\
{\left[\left(u_{-}(1+L)\right)^{1 / 3}\right]^{2} } & =(\rho(L))^{2 / 3} \mathrm{e}^{i\left(-\frac{\pi}{3}-\frac{2}{3} \varepsilon(L)\right)} .
\end{aligned}
$$

Finally, notice that

$$
\lim _{L \rightarrow+\infty} \varepsilon(L)=0 \quad \text { and } \quad \rho(L) \sim \sqrt{L}, \quad L \rightarrow+\infty .
$$

Hence, combining (3.9), (3.10) and (3.11), we obtain

$$
\lim _{L \rightarrow+\infty} \frac{4}{27} \phi(1+L)=\lim _{L \rightarrow+\infty} \frac{3}{\mathrm{e}^{i\left(\frac{2}{3} \varepsilon(L)\right)}+\mathrm{e}^{-i\left(\frac{2}{3} \varepsilon(L)\right)}+1}-1=0 .
$$

Since $\phi$ is analytic in a neighborhood of infinity, then also $\lim _{z \rightarrow \infty} \phi(z)=0$.

Now we give the proof of Theorem 3.1

Proof of Theorem 3.1. It is enough to prove the cases $\alpha=0$ and $\alpha=1$. The more general case $\alpha>0$ is then obtained by taking $\tilde{P}_{n, N}(z):=P_{n, N}(\alpha z) / \alpha^{n}$.

We first prove the case $\alpha=1$. By the assumptions on the zeros of the polynomials $P_{n, N}$ every member of

$$
\left\{\frac{P_{n, N}(z)}{P_{n+1, N}(z)} \mid n, N \in \mathbb{N}, n \leq t^{*} N\right\}
$$

satisfies the estimate in part (a) of Lemma 3.4. So, the family (3.13) is uniformly bounded on compact subsets of $\overline{\mathbb{C}} \backslash[m, M]$. By the theorem of Montel [17, p. 563] we then know that (3.13) is a normal family on $\overline{\mathbb{C}} \backslash[m, M]$. For a 
sequence $\left\{\left(n_{j}, N_{j}\right)\right\}_{j \geq 1}$, with $n_{j}, N_{j} \rightarrow \infty, n_{j} / N_{j} \rightarrow t$ as $j \rightarrow \infty$, we have that, if $j$ is sufficiently large, the function

$$
f_{j}(z):=\frac{P_{n_{j}, N_{j}}(z)}{P_{n_{j}+1, N_{j}}(z)}
$$

belongs to the normal family (3.13). The corresponding sequence $\left\{f_{j}\right\}_{j \geq 1}$ then has a subsequence that converges uniformly on compact subsets of $\overline{\mathbb{C}} \backslash[m, M]$. If we can prove that the limit of any such subsequence is $\phi$, then, by a standard compactness argument, the full sequence $\left\{f_{j}\right\}_{j \geq 1}$ converges uniformly on compact subsets of $\overline{\mathbb{C}} \backslash[m, M]$ to $\phi$. This then proves the theorem in the case $\alpha=1$.

We will show that for each sequence $n_{i}, N_{i} \rightarrow \infty$ with $n_{i} / N_{i} \rightarrow t$ such that the functions $\left\{f_{i}\right\}_{i \geq 1}$ converge uniformly on compact subsets of $\overline{\mathbb{C}} \backslash[m, M]$, we have

$$
f(z):=\lim _{i \rightarrow \infty} f_{i}(z)=\phi(z)+\mathcal{O}\left(z^{-k}\right), \quad \text { as } z \rightarrow \infty,
$$

for each $k \in \mathbb{N}$. The uniqueness of the Laurent expansion around infinity then implies that $f(z)=\phi(z)$. We show this by induction on $k$. The case $k=1$ follows from Lemma 3.5 (b) and $f_{i}(z)=\mathcal{O}\left(z^{-1}\right)$, for every $i \geq 1$. Next, suppose that the claim holds for some $k \geq 1$ and consider a sequence $\left\{\left(n_{i}, N_{i}\right)\right\}_{i \geq 1}$ such that $n_{i}, N_{i} \rightarrow \infty, n_{i} / N_{i} \rightarrow x$ and the functions $\left\{f_{i}\right\}_{i \geq 1}$ converge uniformly on compact subsets of $\overline{\mathbb{C}} \backslash[m, M]$ to some function $f$ as $i \rightarrow \infty$. If we put

$$
\begin{aligned}
& g_{i}(z):=\frac{P_{n_{i}-1, N_{i}}(z)}{P_{n_{i}, N_{i}}(z)}, \quad z \in \mathbb{C} \backslash[m, M], \\
& h_{i}(z):=\frac{P_{n_{i}-2, N_{i}}(z)}{P_{n_{i}-1, N_{i}}(z)}, \quad z \in \mathbb{C} \backslash[m, M],
\end{aligned}
$$

then from the recurrence relation (3.1) we obtain

$$
z=f_{i}(z)^{-1}+b_{n_{i}, N_{i}}+c_{n_{i}, N_{i}} g_{i}(z)+d_{n_{i}, N_{i}} h_{i}(z) .
$$

Since $t<t^{*}$ we may assume without loss of generality that $n_{i}<t^{*} N_{i}$ for every $i \geq 1$. Then $\left\{g_{i}\right\}_{i \geq 1}$ and $\left\{h_{i}\right\}_{i \geq 1}$ are subsets of the normal family (3.13). Therefore, there is a sequence $i_{j} \rightarrow \infty, j \rightarrow \infty$, such that $\left\{g_{i_{j}}\right\}_{j \geq 1}$ and $\left\{h_{i_{j}}\right\}_{j \geq 1}$ converge uniformly on compact subsets of $\overline{\mathbb{C}} \backslash[m, M]$ with limits $g$ and $h$, respectively. If we pass to such a subsequence and take limits in (3.16), then by (3.2) we find

$$
z=\frac{1}{f(z)}+3\left(\frac{4}{27}\right)+3\left(\frac{4}{27}\right)^{2} g(z)+\left(\frac{4}{27}\right)^{3} g(z) h(z), \quad z \in \mathbb{C} \backslash[m, M]
$$

By the induction hypothesis we now have that

$$
\begin{aligned}
& g(z)=\phi(z)+\mathcal{O}\left(z^{-k}\right), \quad z \rightarrow \infty, \\
& h(z)=\phi(z)+\mathcal{O}\left(z^{-k}\right), \quad z \rightarrow \infty .
\end{aligned}
$$

Applying this to (3.17), by Lemma 3.5 we then get

$$
\frac{1}{f(z)}=z-3\left(\frac{4}{27}\right)-3\left(\frac{4}{27}\right)^{2} \phi(z)-\left(\frac{4}{27}\right)^{3} \phi(z)^{2}+\mathcal{O}\left(z^{-k}\right)=\frac{1}{\phi(z)}+\mathcal{O}\left(z^{-k}\right) .
$$

Since $\phi(z)=\mathcal{O}\left(z^{-1}\right)$, this implies

$$
f(z)=\frac{\phi(z)}{1+\phi(z) \mathcal{O}\left(z^{-k}\right)}=\frac{\phi(z)}{1+\mathcal{O}\left(z^{-k-1}\right)}=\phi(z)+\mathcal{O}\left(z^{-k-2}\right)
$$


So we proved that (3.15) also holds with $k$ replaced by $k+2$. Therefore, it holds for all $k$.

Finally, for the case $\alpha=0$ the proof is similar. In fact, (3.3) then easily follows by taking limits in (3.16).

\section{Proof of Theorem 2.1}

In order to prove the asymptotic result in Theorem 2.1 we first have a closer look at the function $\frac{\phi^{\prime}}{\phi}$, which is analytic on $\mathbb{C} \backslash[0,1]$. Here we will use the relation

$$
\frac{\phi^{\prime}(z)}{\phi(z)}=\frac{\frac{4 \phi(z)}{27}+1}{z\left(\frac{8 \phi(z)}{27}-1\right)}=\frac{1}{2 z}+\frac{3}{2 z}\left(\frac{8 \phi(z)}{27}-1\right)^{-1},
$$

which can be obtained by differentiating part (a) of Lemma 3.5. First of all, we are interested in the jump across its branch cut.

Lemma 4.1. The jump of the function $\frac{\phi^{\prime}}{\phi}$ across its branch cut is given by

$$
m(x):=\lim _{\varepsilon \downarrow 0} \frac{\phi^{\prime}(x+i \epsilon)}{\phi(x+i \varepsilon)}-\lim _{\varepsilon \downarrow 0} \frac{\phi^{\prime}(x-i \varepsilon)}{\phi(x-i \epsilon)}=2 \pi i \frac{\mathrm{d} v_{[0,1]}}{\mathrm{d} x}(x), \quad x \in(0,1),
$$

where $v_{[0,1]}$ is defined as in Theorem 2.1 .

Proof. Let $x \in(0,1)$. By (4.1) we easily obtain

$$
m(x)=\frac{3}{2 x}\left\{\lim _{\varepsilon \downarrow 0}\left(\frac{8 \phi\left(x \mathrm{e}^{i \varepsilon}\right)}{27}-1\right)^{-1}-\lim _{\varepsilon \downarrow 0}\left(\frac{8 \phi\left(x \mathrm{e}^{-i \varepsilon}\right)}{27}-1\right)^{-1}\right\} .
$$

Applying the definitions (3.5) we get

$$
\begin{aligned}
& \lim _{\varepsilon \downarrow 0}\left(-1+\sqrt{1-x \mathrm{e}^{ \pm i \varepsilon}}\right)^{1 / 3}=\mathrm{e}^{i \frac{\pi}{3}}(1 \pm \sqrt{1-x})^{1 / 3}, \\
& \lim _{\varepsilon \downarrow 0}\left(-1-\sqrt{1-x \mathrm{e}^{ \pm i \varepsilon}}\right)^{1 / 3}=\mathrm{e}^{-i \frac{\pi}{3}}(1 \mp \sqrt{1-x})^{1 / 3} .
\end{aligned}
$$

Using the notation $v_{ \pm}(x):=(1 \pm \sqrt{1-x})^{1 / 3}$ we then have

$$
\lim _{\varepsilon \downarrow 0} \frac{8 \phi\left(x \mathrm{e}^{ \pm i \varepsilon}\right)}{27}-1=3 x^{1 / 3}\left\{\mathrm{e}^{-\frac{i \pi}{3}} v_{ \pm}(x)+e^{\frac{i \pi}{3}} v_{\mp}(x)\right\}-3 .
$$

So, also applying the relations $x^{1 / 3}=v_{+}(x) v_{-}(x)$ and $2=v_{+}(x)^{3}+v_{-}(x)^{3}$, equation (4.3) becomes

$$
\begin{aligned}
m(x) & =\frac{2 \sqrt{3} i}{x^{2 / 3}} \frac{v_{+}(x)-v_{-}(x)}{\left(x^{1 / 3}\left(v_{+}(x)+v_{-}(x)\right)-2\right)^{2}+3 x^{2 / 3}\left(v_{+}(x)-v_{-}(x)\right)^{2}} \\
& =\frac{2 \sqrt{3} i}{x^{2 / 3}}\left(v_{+}(x)-v_{-}(x)\right)^{-1}\left[\left(v_{-}(x)^{2}-v_{+}(x)^{2}\right)^{2}+3 v_{+}(x)^{2} v_{-}(x)^{2}\right]^{-1}
\end{aligned}
$$

If we multiply the numerator and denominator both by $v_{+}(x)+v_{-}(x)$, then we finally obtain

$$
m(x)=\frac{2 \sqrt{3} i}{x^{2 / 3}} \frac{v_{+}(x)+v_{-}(x)}{\left(v_{+}(x)^{3}+v_{-}(x)^{3}\right)\left(v_{+}(x)^{3}-v_{-}(x)^{3}\right)}=\frac{\sqrt{3} i}{2 x^{2 / 3}} \frac{v_{+}(x)+v_{-}(x)}{\sqrt{1-x}},
$$

which proves (4.2). 
A second point of interest is the behavior of the function $\frac{\phi^{\prime}}{\phi}$ in the neighborhood of its branch points 0 and 1 .

Lemma 4.2. Near the points 0 and 1 we have

$$
\begin{aligned}
& \left|\frac{\phi^{\prime}\left(\varepsilon \mathrm{e}^{i \theta}\right)}{\phi\left(\varepsilon \mathrm{e}^{i \theta}\right)}\right|=\mathcal{O}\left(\varepsilon^{-2 / 3}\right), \quad \varepsilon \downarrow 0, \quad \theta \in(0,2 \pi), \\
& \left|\frac{\phi^{\prime}\left(1+\varepsilon \mathrm{e}^{i \theta}\right)}{\phi\left(1+\varepsilon \mathrm{e}^{i \theta}\right)}\right|=\mathcal{O}\left(\varepsilon^{-1 / 2}\right), \quad \varepsilon \downarrow 0, \quad \theta \in(-\pi, \pi) .
\end{aligned}
$$

Proof. For $\theta \in(0,2 \pi)$ we easily see that

$$
\lim _{\varepsilon \downarrow 0} \phi\left(\varepsilon \mathrm{e}^{i \theta}\right)=-\frac{27}{4}, \quad\left|\frac{4 \phi\left(\varepsilon \mathrm{e}^{i \theta}\right)}{27}+1\right|=\mathcal{O}\left(\varepsilon^{1 / 3}\right), \quad \varepsilon \downarrow 0 .
$$

Applying this to the first equality in (4.1) then gives expression (4.4).

We now take $\theta \in(-\pi, \pi)$. We then have $u_{ \pm}\left(1+\varepsilon e^{i \theta}\right)=-1 \pm \sqrt{\varepsilon} \mathrm{e}^{i \frac{\pi+\theta}{2}}$, where we use the notation (3.7), and applying the definition of the third root (3.5) we get

$$
\lim _{\varepsilon \downarrow 0} \phi\left(1+\varepsilon \mathrm{e}^{i \theta}\right)=\frac{27}{4}\left(\frac{3 \omega_{3}}{2}\left\{\omega_{3} \mathrm{e}^{\frac{\pi i}{3}}+\mathrm{e}^{-\frac{\pi i}{3}}\right\}-1\right)=\frac{27}{8} .
$$

If we write $u_{ \pm}\left(1+\varepsilon e^{i \theta}\right):=\rho_{ \pm}(\varepsilon, \theta) \mathrm{e}^{i \eta_{ \pm}(\varepsilon, \theta)}$, meaning the polar coordinates, then a closer look gives

$$
\eta_{ \pm}(\varepsilon, \theta)= \pm \pi \mp \sqrt{\varepsilon} \cos (\theta / 2)+\mathcal{O}(\varepsilon), \quad \varepsilon \downarrow 0,
$$

and

$$
\begin{aligned}
& \mathrm{e}^{i \frac{\eta_{ \pm}(\varepsilon, \theta)}{3}}=\mathrm{e}^{ \pm \frac{\pi i}{3}}\left(1 \mp \frac{i \sqrt{\varepsilon}}{3} \cos (\theta / 2)\right)+\mathcal{O}(\varepsilon), \quad \varepsilon \downarrow 0, \\
& \rho_{ \pm}(\varepsilon, \theta)^{1 / 3}=1 \pm \frac{\sqrt{\varepsilon}}{3} \sin (\theta / 2)+\mathcal{O}(\varepsilon), \quad \varepsilon \downarrow 0 .
\end{aligned}
$$

From this we easily obtain

$$
\frac{8 \phi\left(1+\varepsilon \mathrm{e}^{i \theta}\right)}{27}-1=-\sqrt{3 \varepsilon} \mathrm{e}^{\frac{i \theta}{2}}+\mathcal{O}(\varepsilon), \quad \varepsilon \downarrow 0 .
$$

Applying (4.6) and (4.9) to the first equality in (4.1) then finally leads to (4.5).

As a corollary of Lemma 4.1 and Lemma 4.2 we obtain that $\frac{\phi^{\prime}}{\phi}$ is the Stieltjes transform of the measure $v_{[0,1]}$, up to a minus sign.

Lemma 4.3. Let $\phi$ be defined by (3.4); then

$$
\frac{\phi^{\prime}(z)}{\phi(z)}=-\int \frac{1}{z-x} \mathrm{~d} v_{[0,1]}(x), \quad z \in \mathbb{C} \backslash[0,1],
$$

with $v_{[0,1]}$ defined as in Theorem 2.1.

Proof. By Lemma 4.1 and Lemma 4.2 and applying Lemma 3.5 (b) to (4.1), the function $\frac{\phi^{\prime}}{\phi}$ satisfies the following additive Riemann-Hilbert problem:

(P1) $f$ is analytic in $\mathbb{C} \backslash[0,1]$,

(P2) $\lim _{\varepsilon \downarrow 0} f(x+i \varepsilon)-\lim _{\varepsilon \downarrow 0} f(x-i \varepsilon)=2 \pi i \frac{\mathrm{d} v_{[0,1]}}{\mathrm{d} x}(x), \quad$ for $x \in(0,1)$, 
(P3) $f(z)=-z^{-1}+\mathcal{O}\left(z^{-2}\right), \quad$ as $z \rightarrow \infty$,

(P4) $f(z)=\mathcal{O}\left(z^{-2 / 3}\right), \quad$ as $z \rightarrow 0$, $f(z)=\mathcal{O}\left((1-z)^{-1 / 2}\right), \quad$ as $z \rightarrow 1$.

If $f$ and $g$ are both solutions of this Riemann-Hilbert problem, then it is easily seen that $f-g$ is analytic in $\mathbb{C} \backslash\{0,1\}$. Moreover, 0 and 1 are removable singularities by (P4). Liouville's Theorem and (P3) then imply $f \equiv g$, meaning that the RiemannHilbert problem has a unique solution. So it is enough to show that

$$
f(z):=\int \frac{1}{x-z} \mathrm{~d} v_{[0,1]}(x), \quad z \in \mathbb{C} \backslash[0,1],
$$

satisfies $(\mathrm{P} 1)-(\mathrm{P} 4)$. Properties $(\mathrm{P} 1)$ and $(\mathrm{P} 3)$ easily follow from the fact that $\frac{\mathrm{d} v_{[0,1]}}{\mathrm{d} x}$ is a probability measure on $(0,1)$. By the Plemelj-Sokhotskii formula for Cauchy integrals, see, e.g., [23, p. 43, (18.1)], $f$ satisfies (P2). Finally, the behavior at the branch points, see (P4), easily follows from [23, p. 74, (29.5) and (29.6)] and (2.4).

Remark 4.4. As an easy consequence of Lemma 4.3 we obtain

$$
-\int \frac{1}{z-x} \mathrm{~d} v_{[0, \alpha]}(x)=\left\{\begin{array}{ll}
\frac{1}{\alpha} \frac{\phi^{\prime}(z / \alpha)}{\phi(z / \alpha)}, & \alpha>0, \\
-\frac{1}{z}, & \alpha=0,
\end{array} \quad z \in \mathbb{C} \backslash[0, \alpha],\right.
$$

where $v_{[0, \alpha]}$ is defined as in Theorem 2.1]

We are now ready to prove Theorem 2.1 .

Proof of Theorem 2.1. Let $t>0$ and fix a number $t^{\star}>t$. Clearly, the convergence (2.2) and the fact that the function $\alpha$ is continuous on $[0, \infty)$ imply that the recurrence coefficients are uniformly bounded if $n / N$ is restricted to compact subsets of $[0, \infty)$. So,

$$
0<R:=\sup \left\{1+\left|b_{n, N}\right|+\left|c_{n, N}\right|+\left|d_{n, N}\right|: n \leq t^{\star} N\right\}<+\infty .
$$

By the recurrence (2.1) we have $P_{n, N}(z)=\operatorname{det}\left(z I_{n}-L_{n, N}\right)$, with

$$
L_{n, N}=\left(\begin{array}{cccccc}
b_{0, N} & 1 & 0 & \ldots & \cdots & 0 \\
c_{1, N} & b_{1, N} & 1 & \ddots & & \vdots \\
d_{2, N} & c_{2, N} & b_{2, N} & 1 & \ddots & \vdots \\
0 & d_{3, N} & c_{3, N} & b_{3, N} & \ddots & 0 \\
\vdots & \ddots & \ddots & \ddots & \ddots & 1 \\
0 & \ldots & 0 & d_{n-1, N} & c_{n-1, N} & b_{n-1, N}
\end{array}\right) .
$$

As a consequence, the zeros of $P_{n, N}$ are bounded by $\left\|L_{n}\right\|_{\infty}$. For $n \leq t^{\star} N$ we then have that the simple zeros of $P_{n, N}$ lie in the interval $[-R, R]$. Moreover, they are assumed to satisfy the interlacing property (for fixed $N$ ) and one can observe that

$$
\alpha(s t) \leq\left(1+\frac{4 \alpha(s t)}{27}\right)^{3} \leq R, \quad \text { for each } 0<s \leq 1
$$


So, by Remark 3.3 and Remark 4.4 we establish

$$
\lim _{n / N \rightarrow t}\left(\frac{P_{\lfloor s n\rfloor+1, N}^{\prime}(z)}{P_{\lfloor s n\rfloor+1, N}(z)}-\frac{P_{\lfloor s n\rfloor, N}^{\prime}(z)}{P_{\lfloor s n\rfloor, N}(z)}\right)=\int \frac{1}{z-x} \mathrm{~d} v_{[0, \alpha(s t)]}(x)
$$

uniformly on compact subsets of $\mathbb{C} \backslash[-R, R]$, where $0<s \leq 1$ and $\lfloor s n\rfloor$ denotes the greatest integer less than or equal to $s n$.

Note that

$$
\frac{1}{n} \frac{P_{n}^{\prime}(z)}{P_{n}(z)}=\frac{1}{n} \sum_{k=0}^{n-1}\left(\frac{P_{k+1}^{\prime}(z)}{P_{k+1}(z)}-\frac{P_{k}^{\prime}(z)}{P_{k}(z)}\right)=\int_{0}^{1}\left(\frac{P_{\lfloor s n\rfloor+1}^{\prime}(z)}{P_{\lfloor s n\rfloor+1}(z)}-\frac{P_{\lfloor s n\rfloor}^{\prime}(z)}{P_{\lfloor s n\rfloor}(z)}\right) \mathrm{d} s .
$$

For $n \leq t^{\star} N$ the zeros of the polynomials $P_{n, N}$ are simple, lie in $[-R, R]$ and satisfy the interlacing property for fixed $N$. From Lemma 3.4 (b) we then get

$$
\left|\frac{P_{\lfloor s n\rfloor+1, N}(z)}{P_{\lfloor s n\rfloor, N}(z)}\right| \leq 2|z|, \quad|z|>R .
$$

With a similar argument as in Lemma 3.4 (a) we can also prove

$$
\left|\left(\frac{P_{\lfloor s n\rfloor, N}(z)}{P_{\lfloor s n\rfloor+1, N}(z)}\right)^{\prime}\right| \leq \frac{1}{\operatorname{dist}(z,[-R, R])^{2}}, \quad z \in \mathbb{C} \backslash[-R, R] .
$$

Combining these two results we have, for $|z|>R$,

$$
\begin{aligned}
\left|\frac{P_{\lfloor s n\rfloor+1}^{\prime}(z)}{P_{\lfloor s n\rfloor+1}(z)}-\frac{P_{\lfloor s n\rfloor}^{\prime}(z)}{P_{\lfloor s n\rfloor}(z)}\right| & =\left|\frac{P_{\lfloor s n\rfloor+1, N}(z)}{P_{\lfloor s n\rfloor, N}(z)}\right|\left|\left(\frac{P_{\lfloor s n\rfloor, N}(z)}{P_{\lfloor s n\rfloor+1, N}(z)}\right)^{\prime}\right| \\
& \leq \frac{2|z|}{\operatorname{dist}(z,[-R, R])^{2}} .
\end{aligned}
$$

So, we can apply Lebesgue's dominated convergence theorem on (4.15), and by (4.14) we obtain

$$
\begin{aligned}
\lim _{n / N \rightarrow t} \int \frac{1}{z-x} \mathrm{~d} \nu\left(P_{n, N}\right)(x) & =\int_{0}^{1} \int \frac{1}{z-x} \mathrm{~d} v_{[0, \alpha(s t)]}(x) \mathrm{d} s \\
& =\frac{1}{t} \int_{0}^{t} \int \frac{1}{z-x} \mathrm{~d} v_{[0, \alpha(s)]}(x) \mathrm{d} s
\end{aligned}
$$

for $|z|>R$. By [16, Theorem 2], which is a gloss on the theorem of Grommer and Hamburger [31, p. 104-105], we then finally establish (2.3).

\section{REFERENCES}

[1] M. Abramowitz and I. A. Stegun. Handbook of Mathematical Functions. Dover Publications, New York, 1968.

[2] A. I. Aptekarev. Multiple orthogonal polynomials. J. Comput. Appl. Math. 99 (1998), 423447. MR1662713 (99m:42036)

[3] A. I. Aptekarev, P. M. Bleher, and A. B. J. Kuijlaars. Large $n$ limit of Gaussian random matrices with external source, part II. Comm. Math. Phys. 259 (2005), 367-389. MR2172687 (2006f:82036)

[4] A. I. Aptekarev, V. Kalyagin, G. López Lagomasino, and I. A. Rocha. On the limit behavior of recurrence coefficients for multiple orthogonal polynomials. J. Approx. Theory 139 (2006), 346-370. MR2220045 (2007a:42048) 
[5] A. I. Aptekarev, V. Kaliaguine, and J. Van Iseghem. The genetic sums' representation for the moments of a system of Stieltjes functions and its application. Constr. Approx. 16 (2000), 487-524. MR1771693 (2001g:41021)

[6] P. M. Bleher and A. B. J. Kuijlaars. Integral representations for multiple Hermite and multiple Laguerre polynomials. Annales de l'Institut Fourier 55 (2005), 2001-2014. MR 2187942 (2007c:33014)

[7] P. M. Bleher and A. B. J. Kuijlaars. Random matrices with external source and multiple orthogonal polynomials. Internat. Math. Research Notices 2004:3 (2004), 109-129. MR2038771 (2004k:82034)

[8] P. M. Bleher and A. B. J. Kuijlaars. Large $n$ limit of Gaussian random matrices with external source, part I. Comm. Math. Phys. 252 (2004), 43-76. MR2103904 (2005i:82031)

[9] M. G. de Bruin. Simultaneous Padé approximation and orthogonality. In Orthogonal polynomials and applications (C. Brezinski et al., ed.), volume 1171 of Lecture Notes in Mathematics, pages 74-83. Springer, Berlin, 1985. MR838972 (87m:41019)

[10] M. G. de Bruin. Some aspects of simultaneous rational approximation. In Numerical Analysis and Mathematical Modeling, volume 24 of Banach Center Publications, pages 51-84. PWNPolish Scientific Publishers, Warsaw, 1990. MR.1097402 (92b:41030)

[11] A. Bultheel, A. Cuyt, M. Van Barel, B. Verdonk, and W. Van Assche. Generalizations of orthogonal polynomials. J. Comput. Appl. Math. 179 (2005), 57-95. MR2134361(2006a:42034)

[12] Y. Ben Cheikh and N. Ben Romdhane. $d$-orthogonal polynomial sets of Chebyshev type. Preprint

[13] T. S. Chihara. An Introduction to Orthogonal Polynomials. Gordon and Breach, New York, 1978. MR0481884(58:1979)

[14] K. Douak and P. Maroni. On $d$-orthogonal Tchebychev polynomials, I. Appl. Num. Math. 24 (1997), 23-53. MR1454707 (98g:42038)

[15] F. P. Gantmacher and M. G. Krein. Oscillation Matrices and Kernels and Small Vibrations of Mechanical Systems, Revised edition. AMS Chelsea Publishing, Providence, RI, 2002. MR:1908601 (2003f:34161)

[16] J. Geronimo and T. P. Hill. Necessary and sufficient condition that the limit of Stieltjes transforms is a Stieltjes transform. J. Approx. Theory 121 (2003), no. 1, 54-60. MR1962995 (2004a:60040)

[17] P. Henrici. Special functions - integral transforms - asymptotics - continued fractions, volume 2 of Applied and computational complex analysis (reprint of the 1977 original). Wiley Classics Library, Wiley-Interscience Publication, John Wiley \& Sons, Inc., New York, 1991. MR.1164865 (93b:30001)

[18] M. E. H. Ismail. Classical and Quantum Orthogonal Polynomials in One Variable, volume 98 in Encyclopedia of Mathematics and Its Applications. Cambridge University Press, Cambridge, 2005. MR2191786(2007f:33001)

[19] A. B. J. Kuijlaars. Chebyshev quadrature for measures with strong singularity. J. Comp. Appl. Math. 65 (1995), 207-214. MR1379132 (96m:41050)

[20] A. B. J. Kuijlaars and S. Serra Capizzano. Asymptotic zero distribution of orthogonal polynomials with discontinuously varying recurrence coefficients. J. Approx. Theory 113 (2001), no. 1, 142-155. MR.1866252 (2002i:42029)

[21] A. B. J. Kuijlaars and W. Van Assche. The asymptotic zero distribution of orthogonal polynomials with varying recurrence coefficients. J. Appr. Theory 99 (1999), 167-197. MR1696553 (2000h:42015)

[22] K. Mahler. Perfect systems. Compositio Math. 19 (1968), 95-166. MR0239099 (39:458)

[23] N. I. Muskhelishvili. Singular integral equations, Boundary problems of function theory and their application to mathematical physics, Translated from the second (1946) Russian edition and with a preface by J. R. M. Radok. Corrected reprint of the 1953 English translation, Dover Publications, Inc., New York, 1992. MR1215485 (94a:45001)

[24] P. Nevai. Orthogonal polynomials. Mem. Amer. Math. Soc. 213 (1979). MR.519926 (80k:42025)

[25] E. M. Nikishin and V. N. Sorokin. Rational Approximants and Orthogonality, volume 92 in Translations of Mathematical Monographs. Amer. Math. Soc., Providence, RI, 1991. MR.1130396 (92i:30037) 
[26] E. B. Saff and V. Totik. Logarithmic Potentials with External Fields, volume 316 in Grundlehren der mathematischen Wissenschaften. Springer-Verlag, Berlin/NY, 1997. MR:1485778 (99h:31001)

[27] W. Van Assche. Multiple orthogonal polynomials, irrationality and transcendence. In volume 236 of Continued fractions: from Analytic Number Theory to Constructive Approximation, Contemporary Mathematics (B. C. Berndt et al., ed.), pages 325-342. Amer. Math. Soc., Providence RI, 1999. MR1665377 (2000k:42039)

[28] W. Van Assche. Asymptotics for orthogonal polynomials and three-term recurrences. In "Orthogonal Polynomials" (P. Nevai, ed.), volume 294 of NATO ASI Series C, pages 435-462. Kluwer Academic, Dordrecht, 1990. MR:1100305 (92k:42038)

[29] W. Van Assche and E. Coussement. Some classical multiple orthogonal polynomials. J. Comput. Appl. Math. 127 (2001), 317-347. MR1808581 (2001i:33012)

[30] W. Van Assche and S. B. Yakubovich. Multiple orthogonal polynomials associated with Macdonald functions. Integral Transforms Special Funct. 9 (2000), 229-244. MR1782974 (2001e:42034)

[31] A. Wintner. Spektraltheorie der Unendlichen Matrizen. Hirzel, Leipzig, 1929.

Department of Mathematics, Katholieke Universiteit Leuven, Celestijnenlaan 200 B, 3001 Leuven, Belgium

Department of Mathematics, Katholieke Universiteit Leuven, Celestijnenlaan 200 B, 3001 Leuven, Belgium

Department of Mathematics, Katholieke Universiteit Leuven, Celestijnenlaan 200 B, 3001 Leuven, Belgium

E-mail address: walter@wis.kuleuven.be 